Review Article

\title{
Comparative analysis of the effectiveness of the alternatives current treatments in Meniere's disease
}

\author{
Mónica P. Jiménez, Susana G. Pérez, José C. Torres, Carlos M. Zarate, Rubén Beltrán, \\ Fátima F. Figueroa, Felix Osuna, José M. Zepeda*, Carlos A. López
}

School of Medicine, Autonomous University of Guadalajara, Guadalajara, Jalisco, Mexico

Received: 12 January 2022

Revised: 04 February 2022

Accepted: 05 February 2022

\author{
*Correspondence: \\ Dr. Jose M Zepeda, \\ E-mail: josem.zepeda@edu.uag.mx
}

Copyright: $@$ the author(s), publisher and licensee Medip Academy. This is an open-access article distributed under the terms of the Creative Commons Attribution Non-Commercial License, which permits unrestricted non-commercial use, distribution, and reproduction in any medium, provided the original work is properly cited.

\begin{abstract}
The objective is to compare the efficacy of the treatments currently used in Ménière's disease, assessing their advantages and disadvantages. A bibliographic review was carried out with 11 original articles, of which 10 are in English and 1 in Spanish, from 3 years to date in Journal of Vestibular Research, Otology \& Neurotology, European Archives Oto-Rhino-Laryngology, American Journal of Otolaryngology, Laryngoscope Investigative Otolaryngology, Journal of Neurology, Iranian Journal of Otorhinolaryngology, Revista Ciencia y Salud, respectively. The following books were consulted Harrison's Principles of Internal Medicine; Oído, nariz, garganta y cirugía de cabeza y cuello; Diagnóstico y tratamiento en otorrinolaringología. Cirugía de cabeza y cuello; and, Tratado de otorrinolaringología y patología cervicofacial. Medical treatment with dexamethasone, gentamicin, betahistine and diuretics was evaluated, as well as surgical treatment with endolymphatic sac surgery, vestibular neurectomy and labyrinthectomy. Treatment is individualized, however, betahistine is recommended as the first option, followed by IT gentamicin for the control of vertigo.
\end{abstract}

Keywords: Treatment, Otorhinolaryngology, Ménière, Efficacy and comparatives

\section{INTRODUCTION}

Ménière's disease (MD) is an idiopathic disorder of the inner ear characterized by spontaneous recurrent vertigo, fluctuating sensorineural hearing loss (HNS), auditory fullness, and tinnitus. The main clinic is the recurrence of sudden and unexpected attacks of vertigo that are often debilitating and can seriously affect the quality of life..$^{1-5}$.

The etiology is multifactorial, with endolymphatic hydrops as a characteristic sign. However, its relationship with MD is unclear and recent studies suggest that additional factors are needed to provoke the presenting symptoms. It is classified according to the criteria of the American Academy of Otolaryngology and Head and Neck Surgery definite and probable. The diagnosis is exclusively clinical and with complementary examinations of the inner ear. ${ }^{5}$

The goal of treatment in MD is to improve symptoms during acute symptoms, prevent recurrent attacks, and prevent progressive damage to the patient's auditory and vestibular function. First-line treatment includes dietary modifications (salt restriction, caffeine and alcohol intake) but there are no randomized trials to support this, as well as in the drugs proposed for acute attacks (dimenhydrinate and benzodiazepines) and prophylactic therapy (betahistine, beta-blockers and diuretics). When the first-line drug does not control the symptoms, especially vertigo, it is recommended to administer corticosteroids or gentamicin via the intratympanic (IT) route. If medical treatment does not control vertigo, 
surgical measures such as endolymphatic sac surgery, vestibular neurectomy and labyrinthectomy are considered. ${ }^{1,3,5}$

This bibliographic review aims to highlight the advantages and disadvantages of the treatments currently used in the follow-up of MD, in order to provide the most effective clinical or surgical management depending on the case.

\section{THEORETICAL FRAMEWORK}

\section{Epidemiology}

The prevalence varies internationally, but in the United States it ranges from 3.5 to 513 per 100,000 people, with the current estimate of approximately 2 per 1,000 people. There is a ratio of women and men of 1.89:1, respectively. An increase has been observed with advancing age. ${ }^{6}$

\section{Etiology}

The cause of MD remains elusive and has been attributed to anatomical, infectious, immune, and allergic factors. Includes the theory of lymphatic vessels, mechanical obstruction and endolymphatic absorption disorder, as well as that of immune response and ischemia of the ear. 6,7

\section{Pathophysiology}

The focus has been on the endolymphatic duct and sac, based on the increase in endolymphatic fluid due to its impaired reabsorption. The metabolic waste products of the organ of Corti that circulate in the endolymph are reabsorbed in the endolymphatic sac, perivascular fibrosis can alter this function and favor the development of endolymphatic dropsy (EF).,

\section{Clinical picture}

Triad of symptoms that includes SNH accompanied by intolerance to noise, reduced speech discrimination and variation in the magnitude of phonemic regression, attacks of spontaneous recurrent vertigo lasting minutes or hours, and constant or intermittent tinnitus that increases in intensity before or during the episode vertiginous. It can be accompanied by a feeling of fullness in the affected ear, while the acute episode of nausea and vomiting. $1,3,4,8-10$

Persistent vertigo, underlying hearing loss, and tinnitus can cause anxiety and other psychological disorders such as neurosis and / or depression. ${ }^{9}$

\section{Diagnosis}

It is performed based on clinical, detailed anamnesis and physical examination. , $^{7,11}$
Otoscopy first step of the examination, followed by the otoneurological evaluation. At the time of the crisis, the otoscopy should be normal, and if acumetry with tuning forks is performed there will be hearing loss of perception of the affected ear. A spontaneous horizontal nystagmus appears that can be seen without impairing gaze fixation. Hennebert's sign can be found in advanced forms. $^{7}$

Table 1: Diagnostic criteria for MD.

\begin{tabular}{|c|c|}
\hline \multicolumn{2}{|c|}{ Diagnostic criteria for Méniere's disease, 2015} \\
\hline Definitive & $\begin{array}{l}\text { - } 2 \text { or more episodes of spontaneous } \\
\text { vertigo, each lasting } 12 \text { minutes to } 20 \\
\text { hours. } \\
\text { - } \quad \begin{array}{l}\text { Audiometrically documented low to } \\
\text { medium frequency sensorineural }\end{array} \\
\text { hearing loss of the affected ear. } \\
\text { - } \quad \begin{array}{l}\text { Fluctuating aural symptoms (hearing, } \\
\text { tinnitus, fullness) of the affected ear. }\end{array} \\
\text { - Not represented by other vestibular } \\
\text { diagnosis. }\end{array}$ \\
\hline Probable & $\begin{array}{l}\text { - } 2 \text { or more episodes of spontaneous } \\
\text { vertigo, each lasting } 12 \text { to } 24 \text { hours. } \\
\text { - } \quad \begin{array}{l}\text { Fluctuating aural symptoms (hearing, } \\
\text { tinnitus, fullness) of the affected ear. }\end{array} \\
\text { - Not represented by other vestibular } \\
\text { diagnosis. }\end{array}$ \\
\hline
\end{tabular}

Table adapted from American Academy of Otolaryngology-Head and Neck Surgery (AAO-HNS). ${ }^{3}$

\section{Glycerol test}

Glycerol test confirms the clinical diagnosis of MD. Determine if there are fluctuations in hearing. $1.2 \mathrm{ml} / \mathrm{kg}$ of glycerin is administered orally, mixed with a similar amount of natural juice; audiograms are made before ingestion one and three hours later. It is considered positive if there is:

- $15 \mathrm{~dB}$ improvement in tonal threshold at $\geq 1$ frequency (250 to $4000 \mathrm{~Hz})$.

- $10 \mathrm{~dB}$ improvement in the tonal threshold of 2 or 3 frequencies (125 to $2000 \mathrm{~Hz}$ ).

- $12 \%$ improvement in speech discrimination. ${ }^{8}$

\section{Electrococleography}

Electrococleography elongated sum potentials represent an important characteristic of MD; which is recognized in $85 \%$ of patients. ${ }^{8}$

\section{Medical treatment}

Diet modification primary treatment includes restriction of intake of salt ( $\leq 2000 \mathrm{mg} /$ day), excess water, caffeine and alcohol, as well as adopting the position resting in decubitus in a quiet place during seizures, and preventing 
changes rapid posture. These measures are always followed by drugs, both for acute attacks, as for prophylactic therapy. $2,7,10,12$

Dexamethasone drug that is better absorbed and diffused in the inner ear. Steroid injection is a non-ablative and safe treatment for the management of episodes of vertigo that do not respond to medical therapy, likewise, the acute exacerbation may respond to a short boost with oral steroids. The IT injection controls the symptoms of vertigo and hearing loss. The $4 \mathrm{~g} / 1$ dose injected showed $82 \%$ complete control of vertigo. $3,7,11,12$

In the study by Garduño-Anaya, from the year 2005, a comparison was made of the use of steroids vs placebo, the results suggest that steroids may be more beneficial. Corticosteroids have a lower risk of hearing damage, but less effective in controlling attacks of vertigo compared to gentamicin, which has a potential risk of hearing loss. ${ }^{1,9}$

In a study of 69 patients, 36 were treated with IT dexamethasone $(4 \mathrm{mg} / \mathrm{dl})$ and 33 with IT methylprednisolone $(40 \mathrm{mg} / \mathrm{dl})$. Initially, vertigo control was $75 \%$ and $66 \%$ for dexamethasone and methylprednisolone, respectively. But, after 6 months the efficacy was reduced in both groups. While vertigo control returned to the previous level, in the dexamethasone group after 6 months it remained higher than the pre- treatment level in the methylprednisolone group. ${ }^{14}$

Gentamicin IT minimally invasive outpatient procedure. It reduces vertigo, but impairs the function of the hair cells of the vestibular terminal organs and can affect hearing function. It aims to reduce the incidence and severity of vertigo attacks, as well as the ablation of vestibular function. Its toxicity causes it to be absorbed in the inner ear through the round window, accumulating in type 1 vestibular hair cells and causing atrophy of these and the entire neuroepithelium, decreasing the production of endolymph. An adequate dose reduces HE and inhibits the transmission of vestibular pathological excitation to the central nervous system by selective destruction of the hair cells of the ampulla and oocysts, this method is called chemical labyrinthectomy. $3,6,7,8,15$

The IT injection is normally $0.75 \mathrm{ml}(60 \mathrm{mg})$ at a rate of $40 \mathrm{mg}$ per $\mathrm{ml}$, leaving the patient supine with the ear up for 45 minutes and instructed to swallow as little as possible. The adverse effect is hearing loss. It is discontinued if nystagmus, decreased hearing, or increased instability occur. Control of vertigo is almost always achieved if vestibular function is eliminated, but the risk of hearing loss increases with increasing total dose and frequency. Recent studies with monthly injections have found a vertigo control of $90 \%$ with hearing loss of $17 \%(<10 \mathrm{~dB}) .^{6-8}$

A study conducted in May 2020 concluded that patients receiving gentamicin are 3 times more likely to control vertigo compared to those treated with steroids. In terms of hearing improvement, patients who received gentamicin had a $0.31 \%$ probability of hearing improvement compared to those who were treated with steroids. ${ }^{6}$

Another 2008 Postema study compared Gentamicin vs placebo, showing $10 \mathrm{~dB}$ more hearing loss on average associated with IT gentamicin. ${ }^{9}$

Therefore, different studies report that complete control of vertigo with IT gentamicin is $75-82 \%$, and hearing impairment is marginal. ${ }^{7,8,12}$

Betahistine potent $\mathrm{H} 3$ antagonist and partial H1 agonist, it produces vasodilation at the capillaries, arterioles and arteriovenous arches of the stria vascularis and the spiral ligament. It is recommended to start with doses of $16 \mathrm{mg}$ every 8 hours, and adjust the dose according to evolution. $^{12,15}$

In a study of 361 patients with DM who received standardized conservative treatment, consisting of betahistine $12 \mathrm{mg}$ and hydrochlorothiazide $25 \mathrm{mg}$ for at least six months, it was concluded that vertigo is not effectively controlled. ${ }^{3}$

Diuretics drugs most used for this disorder can have suppressive effects on vertigo and its frequency, and the progression of hearing loss, since they regulate 1 the homeostasis of the inner ear, eliminating water overload. The most used are acetazolamide in doses of $250 \mathrm{mg} 2$ or 3 times a day isosorbide $90 \mathrm{ml} /$ day, glycerol $10 \%$ with sodium chloride $0.9 \%, 0.5 \mathrm{~g} / \mathrm{kg} \mathrm{ml}$ once daily, and hydrochlorothiazide $50 \mathrm{mg}$ daily for one month with dose reduction by function of evolution. Hydroelectrolyte controls should be carried out frequently and, in most cases, supplemented with potassium. . $^{2,311,12}$

Isosorbide, an osmotic diuretic considered as a treatment option to prevent the recurrence of vertigo attacks within 6 months after the last vertigo attack, demonstrating a reduction in $\mathrm{HE}$ without rebound. ${ }^{11,15}$

Glycerol, an osmotic diuretic, capable of dehydrating endolymphatic hydrops of the inner ear by raising the osmotic pressure of the fluid in which it dissolves, which favors the diffusion process and increases plasma osmolarity in the cochlear capillaries and the vascular stria, which, in turn, causes the diffusion of endolymph in the blood circulation reducing the pressure of the membranous labyrinth. However, it is only done once for diagnostic purposes. May have negative systemic effects on kidney and liver function, and electrolyte balance; monthly blood tests are recommended. ${ }^{2}$

In a study carried out in 40 patients at the University of Salerno between 2016 and 2018, it was observed that intravenous infusion of glycerol improved vertigo attacks and reduced the discomfort generated by tinnitus, 
increasing the quality of life in patients with unilateral DM who did not respond to dietary restrictions. ${ }^{2}$

\section{Surgical treatment}

When medical treatment fails to control vertigo, endolymphatic sac surgery, vestibular neurectomy, and labyrinthectomy are considered. Although vestibular neurectomy has a very high rate of vertigo control, the risk is high. Labyrinthectomy is reserved only for patients with total deafness. ${ }^{3}$

Non-ablative surgical options include decompression and bypass of the endolymphatic sac. Studies show that there are no significant differences in hearing changes between these options, however, endolymphatic sac surgery is considered as a prophylaxis of the recurrence of vertigo attacks and the progression of hearing loss before destructive surgery. ${ }^{10}$

Selective destruction of the vestibule is used as a prophylaxis of recurrent vertigo attacks in cases refractory to treatments, including IT gentamicin. ${ }^{9,11}$

\section{DISCUSSION}

In the present bibliographic review, information about DM was analyzed, which was obtained and collected from various sources represented by articles and books, in order to compplow efficacy into current treatment alternatives.

Treatment is aimed at preventing osmotic changes in endolymph. In the course of 20 years to date, drugs such as vasodilators and diuretics have been used, which are useful in these cases. First-line treatment usually includes dietary modifications followed by drugs for both acute attacks (dimenhydrinate, benzodiazepines) and prophylactic therapy (betahistine, beta-blockers, diuretics). Initially the main goal of treatment in the acute period is the remission of dizziness and nausea during attacks and improvement of hearing loss, and the latter is the prevention of attacks of vertigo by administering fluids intravenously during the stay in the emergency room. Medications for vertigo include antihistamines, benzodiazepines and anticholinergics; $7 \%$ sodium bicarbonate intravenous infusion is also effective in reducing vestibular symptoms. Antiemetic drugs include metoclopramide and domperidone. Acute sensorineural hearing loss associated with vertigo attacks is treated with steroids, which are administered orally or intravenously, which generally have a good response and symptom management, which is why it is a point of good practice. Faced with the failure of the response to the medications or the severity of the disease coupled with the start of first-line treatment, it is of utmost importance to implement changes in the patient's lifestyle since stress, fatigue and lack of sleep have some association with the pathogenesis of DM and exacerbation of symptoms, recommending reducing stress by avoiding overwork and unpleasant or compulsory activities, in addition to performing aerobic exercises to reduce the number of attacks and improve hearing loss. ${ }^{4,9,11}$

Previously, a mastoid endolymphatic shunt was used in the endolymphatic sac to decompress excess endolymph, or to perform a selective vestibular nerve. In cases where the vertigo is disabling and there is no longer useful hearing, there is effective control of the vertigo with labyrinthectomy, and destruction of the neural elements. Nowadays it is a question of not resorting to such invasive processes most of the time, especially in elderly patients who do not want to risk this type of process, for which the injection of IT gentamicin is chosen, which has proven to be effective and safe, the current trend being the use of low doses in single applications with patient monitoring to assess the eventual need to repeat the intervention. In some cases, direct placement over the round window by tympanotomy is presented as an alternative injection strategy and, finally, Endolymphatic sac surgery could be an option to consider when other treatments have failed, however, the current available evidence is not enough to recommend it routinely. The current evidence is insufficient to recommend a single treatment; the therapeutic options must be evaluated under the individual criteria of each patient. In all cases, good communication between the physician and the patient is essential for a multidisciplinary approach to achieve successful management. In multiple articles, vertigo improvement results were demonstrated by administering gentamicin with IT steroids, obtaining a superior result with the first drug, which guides us that treating a patient with said medication is favorable. Hair cells are more sensitive to certain drugs, such as aminoglycosides (gentamicin and streptomycin) with cochlear hair cells. It is administered through a ventilation tube or injected into the middle ear, as gentamicin's toxicity makes it possible for its IT injection to destroy dark cells, reducing endolymph production. An adequate dose can reduce $\mathrm{HE}$ and inhibit the transmission of vestibular pathological excitation to the central nervous system through the selective destruction of the hair cells of the ampulla and oocysts, this method is called chemical labyrinthectomy. The side effect of IT gentamicin is hearing loss. ${ }^{7,8,12}$

Intratympanic steroids (STIs) can control vertigo, seizures with a minimized risk of hearing loss and posttreatment imbalance. Studies have shown that this efficacy is associated with inflammation, the processes immunological and the regulation of ionic homeostasis. In another study, the dynamics were presented in vivo patients with internal ear disease where the total volume of HE prior to treatment was significantly correlated with the duration of the disease of the cochlear symptoms and the level of hearing of the affected ear. In DM patients it developed longitudinally with deterioration of inner ear function during medical treatment. The natural course of the disease can progress with the development of HD for at least a certain period. Vertigo episodes improve, but 
both vestibular and auditory function progress to deteriorate. Surgery in the endolymphatic sac improves drainage and reduces dropsy, it is another option commonly performed for intractable patients. Therefore, HD is widely recognized as a typical marker of DM, although this hypothesis has not been conclusively proven, suppression of vertigo after sac surgery was not always the result of reduction, this suggests that this sign increased after Surgery. The therapeutic benefit of betahistine in vertigo symptoms was also shown in a meta-analysis. However, post-treatment HE developed longitudinally in this study, although its volume according to MRI did not change in some patients and continued to develop in others. These findings suggest that HE does not decrease during medical treatment, which is a new concept that conflicts with current knowledge about DM. ${ }^{8,15}$ Although this hypothesis has not been conclusively proven, suppression of vertigo after sac surgery was not always the result of reduction, this suggests that this sign increased after surgery. The therapeutic benefit of betahistine in vertigo symptoms was also shown in a meta-analysis. However, posttreatment HE developed longitudinally in this study, although its volume according to MRI did not change in some patients and continued to develop in others. These findings suggest that $\mathrm{HE}$ does not decrease during medical treatment, which is a new concept that conflicts with current knowledge about DM..$^{8,15}$

Furosemide has a powerful and rapid diuretic effect, which should have a lowering effect on HD. Therefore, we suggest that HD and cofactors are responsible for the symptoms of DM. Although the episodes of vertigo improved superficially during the medical treatment; underlying $\mathrm{HE}$ has developed in vestibular impairment and hearing function impairment. ${ }^{6,8,15}$

\section{CONCLUSSION}

Treatment of MD involves the management of acute attacks and endolymphatic dropsy, on the one hand, and prophylaxis of these attacks with maintenance treatment on the other. For the management of seizures, vestibular sedatives such as dimenhydrinate or diazepam are used for a short period of time, which suppress the sensation of movement. Preventing the appearance of individual seizures is more complex and the therapeutic options are variable, changes in lifestyle, mainly with a low sodium diet, must still be advised, although current evidence is still insufficient to recommend a single treatment, the different therapeutic options available must be used under criteria evaluating the individual situation of each patient.

With regard to medical treatment, betahistine is recommended as the first option, in doses not less than 48 $\mathrm{mg} / \mathrm{day}$, for 6-12 months. But, given the failure of medical treatment, IT gentamicin demonstrated a statistically significant improvement in vertigo with a result that is superior to the rest of the treatments in an effective and safe way, in low doses and single applications with follow-up for the eventual evaluation in the event of the need to repeat the intervention.

Finally, endolymphatic sac surgery could be an option to consider when all other treatments have failed, however, the current available evidence is not sufficient to recommend it routinely.

Although current evidence is insufficient to recommend a single treatment, the different available therapeutic options must be used judiciously, assessing the individual situation of each patient.

\section{Funding: No funding sources \\ Conflict of interest: None declared \\ Ethical approval: Not required}

\section{REFERENCES}

1. Scarpa A, Ralli M, Cassandro C, Gioacchini FM, Alicandri-Ciufelli $\mathrm{M}$, Viola $\mathrm{P}$ et al. Low-dose intratympanic gentamicin administration for unilateral Meniere's disease using a method based on clinical symptomatology: Preliminary results. American journal of otolaryngology. 2019;40(6):102289.

2. Scarpa A, Cassandro C, De Luca P, Greco A, Chiarella G, de Vincentiis $M$ et al. Therapeutic role of intravenous glycerol for Meniere's disease. Preliminary results. American journal of otolaryngology. 2020;41(4):102498.

3. Zhang D, Lv Y, Han Y, Li Y, Li X, Wang J et al. Long-term outcomes of triple semicircular canal plugging for the treatment of intractable Meniere's disease: A single center experience of 361 cases. J Vestib Res. 2019;29(6):315-22.

4. Scarpa A, Ralli M, Viola P, Cassandro C, AlicandriCiufelli M, Iengo $\mathrm{M}$ et al. Food-induced stimulation of the antisecretory factor to improve symptoms in Meniere's disease: our results. Eur Arch Otorhinolaryngol. 2020;277(1):77-83.

5. Egea SA Miranda ME, Salas ES. Enfermedad de Ménière en adultos. Revista Ciencia y Salud. 2020;4(4):96.

6. Jiang M, Zhang Z. Zhao C. What is the efficacy of gentamicin on the incidence of vertigo attacks and hearing in patients with Meniere's disease compared with steroids? A meta- analysis. Journal of Neurology. 2020;7(8).

7. Johnson J, Lalwani AK. Enfermedades vestibulares. Lalwani A.K. 4ta ed. Diagnóstico y tratamiento en otorrinolaringología. Cirugía de cabeza y cuello, 3e. McGraw Hill. 2018.

8. Escajadillo JR. Oído, nariz, garganta y cirugía de cabeza y cuello. México, D.F.: Manual Moderno. 2014.

9. Ahmadzai N, Cheng W, Kilty S, Esmaeilisaraji L, Wolfe D, Bonaparte $\mathrm{J}$ et al. Pharmacologic and surgical therapies for patients with Meniere's 
disease: A systematic review and network metaanalysis. PloS one. 2020;15(9):e0237523.

10. Harrison. Principios de Medicina Interna. 20 ed. McGraw-Hill Education. 2018.

11. Iwasaki S, Shojaku H, Murofushi T, Seo T, Kitahara $\mathrm{T}$, Origasa $\mathrm{H}$ et al. Diagnostic and therapeutic strategies for Meniere's disease of the Japan Society for Equilibrium Research. Auris Nasus Larynx. 2021;48(1):15-22.

12. Alegría JB. Tratado de otorrinolaringología y patología cervicofacial. 1ra ed. Valencia: Elsevier. 2009.

13. Beyea JA, Instrum RS, Agrawal SK, Parnes LS. Intratympanic dexamethasone in the treatment of Meniere's disease: a comparison of two techniques. Otol Neurotol. 2017;38(6):e173-8.
14. Masoumi E, Dabiri S, Ashtiani MTK, Erfanian R, Sohrabpour S, Yazdani N et al. Methylprednisolone versus Dexamethasone for Control of Vertigo in Patients with Definite Meniere's disease. Iranian journal of otorhinolaryngology. 2017;29(95):341.

15. Fukushima $M$, Kitahara $\mathrm{T}$, Oya R, Akahani S, Inohara $\mathrm{H}$, Naganawa $\mathrm{S}$. Longitudinal UpRegulation of Endolymphatic Hydrops in Patients with Meniere's Disease During Medical Treatment. En: The Triological Society. 2017;11:12-4.

Cite this article as: Jiménez MP, Pérez SG, Torres JC, Zarate CM, Beltrán R, Figueroa FF et al. Comparative analysis of the effectiveness of the alternatives current treatments in Meniere's disease. Int J Otorhinolaryngol Head Neck Surg 2022;8:292-7. 